\title{
Elastic $d^{12} C$ scattering within a three-body model
}

\section{Yuriy Uzikov*}

Joint Institute for Nuclear Researches, Dubna, 141980, Russia

Department of Physics, M.V. Lomonosov Moscow State University, RU 11991 Moscow, Russia

Dubna State University, Dubna, 141980 Russia

E-mail: uzikovejinr.ru

Elastic $d^{12} C$ scattering is considered on the basis of the sum of two mechanisms, single and double $N^{12} C$ scattering. The refined Glauber theory is used including full spin dependence of $N^{12} C$ - scattering amplitudes which are produced within the optical model of the elastic $N^{12} C$ scattering. Numerical results are obtained at the deuteron beam energy $270 \mathrm{MeV}$ and compared with the data on unpolarized differential cross section, vector $A_{y}$ and tensor $A_{y y}$ analyzing powers.

23rd International Spin Physics Symposium - SPIN2018 -

10-14 September, 2018

Ferrara, Italy

* Speaker. 


\section{Introduction}

An interest to elastic scattering of polarized deuterons off the ${ }^{12} \mathrm{C}$ nucleus is motivated by planned measurements of electric dipole moment (EDM) of the deuteron. Non-zero intrinsic EDM of particles will indicate directly to time-reversal invariance violation (or CP violation under CPT symmetry) as well as P-parity violation. Knowledge of this signal is important to explain the observed matter-antimatter asymmetry in the Universe. Measurement of EDM of protons and deuterons is planned at COSY (Jülich) by JEDI Collaboration using the COSY storage ring [1]. Interaction of the deuteron EDM with electric field would generate a spin rotation generating additional component of the deuteron polarization vector. This component can be detected by highly sensitive polarimeter with the carbon target [2] in the process of deuteron scattering on carbon nuclei. An important question is to choose an optimal energy of the deuteron beam which would provide a maximal figure of merit. Existing experimental data on polarized $d^{12} C$ elastic scattering in region of $100-200 \mathrm{MeV}$ not enough complete. Therefore theoretical calculations are desirable as a guide for a choose of the preferable beam energy. We consider $d-{ }^{12} C$ system as a three-body $p-n-{ }^{12} C$ system [3] neglecting excitation of the ${ }^{12} C$ nucleus and apply the Glauber theory [4] for calculation of spin observables of the $d^{12} C$ elastic scattering.

\section{The model}

The spin formalism used here for $d^{12} C$ elastic scattering is based on the formalism developed in Ref. [5] for $p d$-elastic scattering within the Glauber. The formalism includes total spin dependence of $p N$-scattering amplitudes and $S$ and $D$ components of the deutreon wave function. This formalism after proper modification was applied to calculations of analyzing powers in antiprotondeuteron elastic scattering [6], spin-correlation coefficients in $p d$ scattering [7] and further developed to account for T-invariance violation effects in proton-deuteron $[8,9]$ and antiproton- deuteron [10] double polarized scattering. The formalism [5] is formulated in non-Madison reference frame and therefore could not be directly applied to an analysis of existing experimental data $[11,12]$ on pd elastic scattering obtained at $135-250 \mathrm{MeV}$, that are presented in the Madison coordinate system [13]. Transformation of the formalism [5] to the Madison frame was done in Refs. [7, 14]. The spin of the ${ }^{12} \mathrm{C}$ nucleus is equal to zero, therefore spin structure of the amplitude of the elastic scattering $d^{12} \mathrm{C} \rightarrow d^{12} \mathrm{C}$ is simpler than the corresponding structure the $p d \rightarrow p d$ amplitude. In particular case, if the $N^{12} C$ amplitudes are taken instead of the $p N$ amplitudes in the process $p d \rightarrow p d$ then the $p d \rightarrow p d$ amplitude transforms to the $d^{12} \mathrm{C} \rightarrow d^{12} \mathrm{C}$ amplitude.

As the first step, the elementary $N^{12} C$ elastic scattering amplitudes are taken here [3] from the optical model. The amplitude of the elastic $N^{12} \mathrm{C}$-scattering contains two independent terms

$$
M_{N}\left(\mathbf{p}, \mathbf{q} ; \sigma_{N}\right)=A_{N}+i B_{N} \sigma_{N} \hat{\mathbf{n}}
$$

here $\sigma_{N}$ is the Pauli matrix acting on the spin state of the nucleon $N(N=p, n)$ in the deuteron, $\hat{\mathbf{n}}$ is the unit vector directed along the vector $\left[\mathbf{p} \times \mathbf{p}^{\prime}\right]$, where $\mathbf{p}\left(\mathbf{p}^{\prime}\right)$ is the momentum of the initial (final) deuteron. The complex amplitudes $A_{N}$ and $B_{N}$ are calculated within the optical model described in Ref. [15] by fit to the data on differential cross section of the elastic $p^{12} \mathrm{C}$ - scattering [16]. 
As a function of the transferred momentum $\mathbf{q}=\mathbf{p}-\mathbf{p}^{\prime}$ the amplitude $A_{N}(q)$ has the $s$ - wave behaviour, whereas the amplitude $B_{N}(q)$ demonstrates the $p$-wave behavior. These amplitudes were parameterized by expansion into Gaussians

$$
\begin{array}{r}
A_{N}(q)=\sum_{i}^{n} C_{i} \exp \left(-a_{i} q^{2}\right), \\
B_{N}(q)=q \sum_{i}^{n} D_{i} \exp \left(-b_{i} q^{2}\right),
\end{array}
$$

where coefficients $C_{i}, D_{i}, a_{i}$ and $b_{i}$ are given in Ref. [3] for the proton beam energy $135 \mathrm{MeV}$ that corresponds to the deuteron beam energy $270 \mathrm{MeV}$. The differential cross section $d \sigma_{N} / d \Omega$ and vector analyzing power $A_{y}$ of the $N^{12} C$-elastic scattering are connected with the amplitudes $A_{N}$ and $B_{N}$ as

$$
\begin{aligned}
d \sigma_{N} / d \Omega & =\left|A_{N}\right|^{2}+\left|B_{N}\right|^{2}, \\
A_{y} & =\frac{2 \operatorname{Im} A_{N} B_{N}^{*}}{\left|A_{N}\right|^{2}+\left|B_{N}\right|^{2}} .
\end{aligned}
$$

The results of calculations for the vector analyzing power $A_{y}^{p}$ of the elastic $p^{12} C$ scattering performed at $135 \mathrm{MeV}$ using these amplitudes are shown in Fig.2, $\mathrm{b}$ in comparison with the data [16].
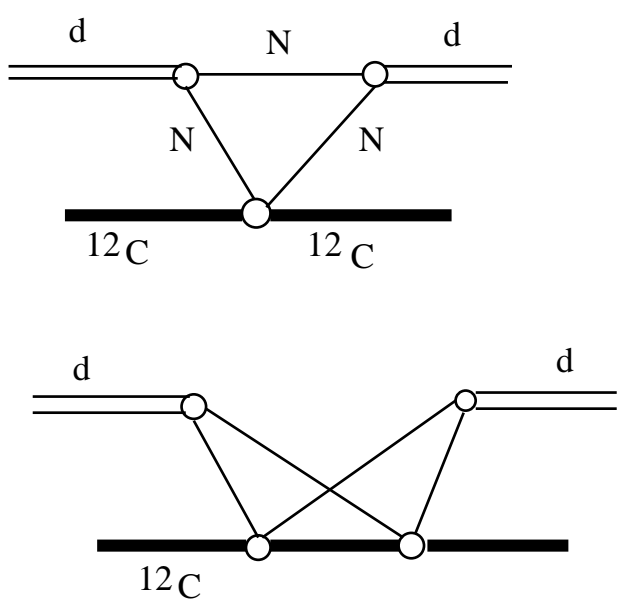

SS

\section{DS}

Figure 1: Single scattering (SS) and double scattering (DS) mechanisms of the $d^{12} C$ elastic scattering

The results of calculations of the observables for the $d^{12} C$ elastic scattering at deuteron beam energy $270 \mathrm{MeV}$ are shown in Fig.2 in comparison with the data [17]. One can see from Fig. 2, $a$ that the SS-mechanism alone describes the data on the differential cross section of the $d^{12} C$ elastic scattering not perfectly, but the coherent sum of the SS and DS mechanisms is in good agreement with this data [17] in forward hemisphere $\theta_{c m} \leq 25^{\circ}$. Another situation occurs for the spin observables $A_{y}^{d}$ and $A_{y y}$. The SS mechanism predicts a peak of $A_{y}^{d}(\theta)$ at $\sim 15^{\circ}$, that is shifted to larger scattering angles by $\sim 3-4^{\circ}$ from the peak observed at the experiment [17]. Inclusion of the DS mechanism properly shifts the peak towards smaller scattering angles but diminishes its magnitude by factor of two lower than the data. On the other hand, the second peak predicted by the 
SS mechanism at $30^{\circ}$ increases after inclusion of the DS mechanism that improves the agreement with the data. For the tensor analyzing power $A_{y y}$ inclusion of the SS mechanism also improves to some extent the agreement with data, but as in the case of $A_{y}^{d}$ underestimates the data by factor of $\sim 2$. One should note that the used here optical model [15] underestimates the $A_{y}^{p}$ in elastic $p^{12} C$ scattering (Fig.2, $b$ ). A similar disagreement between optical model and the data on $A_{y}^{n}$ was found in Ref. [16].
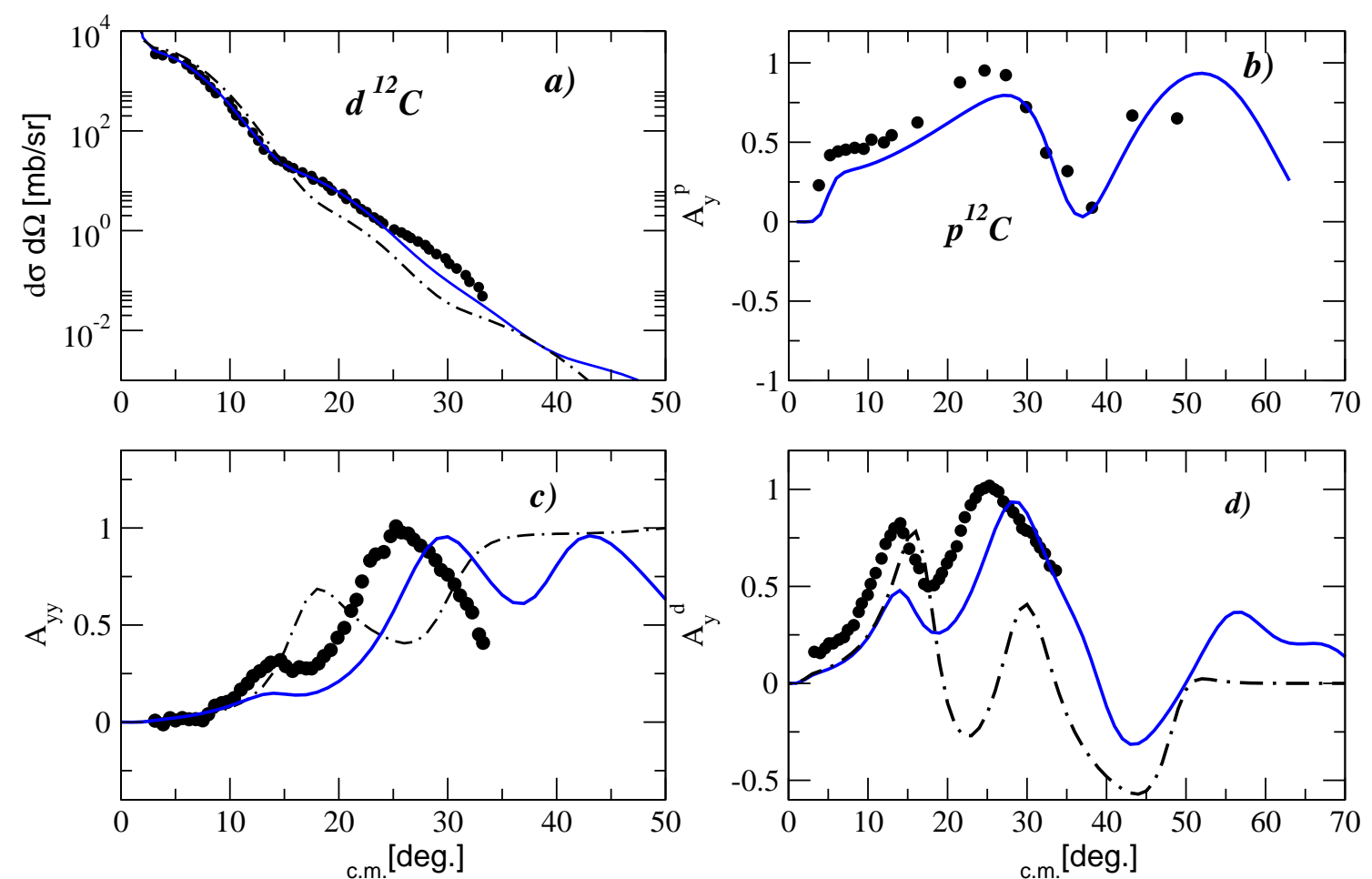

Figure 2: Panels $a, c, d$ : The differential cross section $(a)$, tensor analyzing power $A_{y y}(c)$ and vector analyzing power $A_{y}^{d}(d)$ of the elastic $d^{12} C$ scattering at the deuteron beam energy $270 \mathrm{MeV}$ as functions of the deuteron scattering angle. The lines show the results of calculations within the refined Glauber model (see text) taking into account the single scattering mechanism (dashed-dotted) and coherent sum of the single and double scattering mechanisms (full). Experimental data (•) are taken from [17]. Panel $b$ : The vector analyzing power $A_{y}^{p}$ of the $p^{12} C$ elastic scattering at the proton beam energy $T_{p}=135 \mathrm{MeV}$ versus the $\mathrm{cms}$ scattering angle. The line is the result of the optical model [15]. Experimental data $(\bullet)$ are taken from [16].

\section{Conclusion}

We find [3] that the calculated within the combined Glauber and optical model differential cross section is in a good agreement with the data [17] in the forward hemisphere, whereas the calculated spin observables $A_{y}$ and $A_{y y}$ are only in qualitative agreement with the data. The latter indicates an importance of further development of the model for the amplitudes of the $N^{12} C$ elastic scattering. One possible way is to use the microscopic Glauber model to describe the data on $p^{12} C$ elastic scattering.

Acknowledgements. I am thankful to E. Stephenson for interest to this work. 


\section{References}

[1] G. Guidoboni et al. (JEDI Collab.), Phys. Rev. Lett. 117, 054801 (2016).

[2] N.P.M. Brantjes et al., Nucl. Instr. Methods Phys. Rev. Sect. A 664, 49 (2012).

[3] E. T. Ibrayeva, Yu.N. Uzikov, Yad. Fiz. 81, 451 (2018) [Phys. At. Nucl. 81, 479 (2018)].

[4] R. J. Glauber, Phys. Rev. 100, 242 (1955).

[5] M.N. Platonova and V.I. Kukulin, Phys. Rev. C 81, 014004 (2010); 94, 069902 (Erratum) (2016).

[6] Yu.N. Uzikov and J. Haidenbauer, Phys. Rev. C87, 054003 (2013); Phys. Rev. C 88, 027001 (2013).

[7] A.A. Temerbayev, Yu.N. Uzikov, Yad.Fiz.78, 38 (2015) [Phys. At. Nucl. 78, 35 (2015)].

[8] Yu.N. Uzikov and A.A. Temerbayev, Phys. Rev. C 92, 014002 (2015).

[9] Yu.N. Uzikov, Phys. Part. Nucl. 49, 576 (2018).

[10] Yu. Uzikov, J. Haidenbauer, Eur. Phys.J. Web Conf. 181, 01015 (2018).

[11] K. Sekiguchi et al., Phys. Rev. C 65, 034003 (2002).

[12] B. von Przewoski et al., Phys. Rev. C 74, 064003 (2006).

[13] H. H. Barschall and W. Haeberli, in Proceedings of the Third International Symposium Polarization Phenomena in Nuclear Reactions, Madison, 1970, (Madison, 1970), p. 1.

[14] A.A. Temerbayev, Yu.N. Uzikov, Izv. Ross. Akad. Nauk, Ser.Fiz. 81, 814 (2017) [Bull.Russ.Acad.Sci.Phys. 81, 739 (2017)].

[15] V. Karpov et al., Yad. Fiz. 79, 520 (2016) [Phys. At. Nucl.79, 749(2016)].

[16] J.L. Comfort, and B.C. Karp, Phys. Rev. C 21, 2162 (1980).

[17] Y. Satou et al., Phys. Lett. B 549, 307 (2002). 